\title{
Dynamics of Automatic and Controlled Visual Attention
}

\section{Erich Weichselgartner and George Sperling}

The time course of attention was experimentally observed using two kinds of stimuli: a cue to begin attending or to shift attention, and a stimulus to be attended. Precise measurements of the time course of attention show that it consists of two partially concurrent processes: a fast, effortless, automatic process that records the cue and its neighboring events; and a slower, effortful, controlled process that records the stimulus to be attended and its neighboring events.

A N ESSENTIAL COMPONENT OF VISUal attention is attentional gating $(I)$, the process whereby some incoming information is selected for further analysis or for memorization, while other infornation is ignored or attenuated and lost (2-8). Normally, eve movements and attentional shifts are tightly coupled. In this report we discuss the attentional processes that can occur while the eves are stationary $(9,10)$.

Early in our investigations of the dynamics of attentional gating, we noticed curious bimodal distributions of attention shift times that suggested that we were observing not mercly a single act of attention but two consccutive, partially overlapping acts. During the investigation of these phenomena (II), we learned how to attain scparate, and almost independent, control of the time course of cach attentional process. The first process is a quick, effortless, automatic process triggered by target detection; the second. is a shower, effortful, controlled process (12) whose latency depends on practice and task difficulty.

The procedure we used to measure attention was based on the rapid serial visual prescntation (RSVP) attention shift paradigm of Sperling and Recres (13). RSVP $(14,15)$ produces many regularly spaced cvents that can be used to resolve the temporal properties of attentive processes. In the Sperling and Reeves procedure, a subject monitored a stream of alphabetic characters flashed one on top of the other to the left of visual fixation for the presence of a target letter. On detection, the subject immediately shifted attention to a numeral stream at visual fixation and attempted to remember the earliest occurring numeral from the numeral strean. Typically, subjects remembered a numeral that occurred 300 to 400

Human Information Processing Laboratory, Psycholog: Department, New York Liniversity, New York, NY 10003 . msec after the target. The onset time of a remembered numeral defined the attentionreaction time. When target difficulty or target probability was altered, the attentionreaction time varied much the same way as the motor-reaction time (I3).

In this investigation we studied an additional attentional process that had completely different propertics, using two procedures. In both procedures, the subject maintaincd visual fixation on a numeral strcam throughout the trial. Procedure l (Fig. la), an adaptation of the RSVP attention shift paradigm, scrved as a control condition. Subjects began a trial by attending to a letter stream to the left of the numeral stream. They were instructed to shift attention from the letter stream to the numeral stream as quickly as possible on detection of the target letter $C$ and to remember the earliest four numerals they could (simultancous with or subsequent to target detection). In procedure 2 (Fig. 1b), the experimental condition, there was only one stream. Subjects fixated a numeral stream in which the target was embedded. Two kinds of targets were used to open the attentional gate: (i) outline square condition (OS), an outlite square around one of the numerals, and (ii) highlighted numeral condition ( $\mathrm{HN}$ ), a brightening of onc of the numerals.

For each trial of both procedures, the target was randomly embedded near the middle of a stream of 25 numerals. At the end of stimulus presentation, subjects typed the earlicst occurring numeral they could remenber as well as the three subsequent ones. The four recalled numerals comprised the response sequence. This recall task enabled us to probe the distribution of attention over a longer time period [and at no additional cost (13)] than would be possible with just one recalled numeral.

Over 10,000 trials were conducted with threc subjects. Representative data from all threc are shown (Fig. 2, a-j). Recall data were analyzed in terms of the estimated probabilities $p\left(t_{j}, k\right)$ of reporting a numeral from frame $j$ (time $t_{j}$ ) in position $k$ of the response. The target occurred at time $t_{0}=0$. We first considered $p\left(t_{j}\right)$, the probability of reporting a stimulus item from time $t_{j}$ anywhere in the response (the envelope curves of Fig. 2):

$$
p\left(t_{j}\right)=\sum_{k=1}^{4} p\left(t_{j}, k\right)
$$

The basic finding from the control experiment is shown in Fig. 2a. The numeral simultancous with the target and the one following it were almost never reported. Reported numerals occurred 200 to 700 msec after the target. The trace of the first reported numeral showed that $50 \%$ of the time it was the numeral occurring $300 \mathrm{msec}$ after the target; the remainder of the time it was the numeral occurring 200 or $400 \mathrm{mscc}$ later. In all significant respects, these data replicated the data of Reeves and Sperling (l). They indicated that detecting the target $C$ in the letter stream to the left of visual fixation and shifting attention to the numeral streatn at visual fixation takes $300 \pm 100$ msec.

Typical data from procedure 2 (target OS) are shown in Fig. 2b. The distribution of the $p\left(t_{j}\right)$ clearly is bimodal. Most numerals were recalled from two separated periods in time, the first mode between 0 and 100 msec after target presentation, the second mode between 300 and $400 \mathrm{msec}$ after target presentation. For all subjects, the
Fig. 1. (a) Illustration of procedure 1 (control cxpcriment) and (b) procodure 2 (main cxperiment). On the face of a cathode-tay tube, 25 frames, composed of letters and numerals in procedure 1 or numerals alone in procedure 2, appear in serial order. Presentarion rate is usually 10 frames per second and sometines 12.5 frames per second; framc duration is 18 msec. With equal probability, 7 to 14 stimulus frames precede the target, and the subject's task is to attempt to report the four carliest occurring numcrals simultancous with or subsequent to the target. In order to noid ambignity in the analysis of the response sequence, numerals in frames -2 to +7 are all different. 
recall probability of the critical numeral (the numeral displayed simultaneously with the target) was well above 0.9. Indeed, all subjects reported that they could achieve high recall of the critical numeral effortlessly; it scemed to "pop out" of the background numerals, frequently enhancing the subsequent numeral as well. From this high level of response (and from more elaborate statistical tests) it was obvious that the bimodality did not result from a mixture of early-mode and late-mode trials: a single, bimodal distribution characterized all the responses.

Subjects claimed to be able to distinguish introspectively between numerals that they remembered occurring with the target and those that seemed to have occurred subsequent to the target (in fact, these sometimes occurred earlier). An extended response procedure was developed to utilize this infor- mation. After reporting the four numerals of the response sequence, subjects further reported whether each one seemed to be perceptually attached to the target. We refer to items judged by subjects to be attached to rhe target as first glinpse, the others as second glimpse.

Subjects had no particular difficulty making first glimpse-second glimpse judgments. First glimpse items were described as being effortlessly acquired; recall of second glimpse items required effort. Experiments with subjects E.W. and S.W. in which these judgments were obtained with a highlighted numeral are shown in Fig, 2, c and d. When the response data were obviously bimodal (E.W.), the glimpse judgment mirrored the bimodality. Indeed, for appropriate conditions, we were able to clearly observe bimodal response data for every subject. How- ever, even when the data were not obviously bimodal (S.W.), the glimpse judgment still revealed the same two underlying processes.

When subjects were asked to make glimpse judgments in procedure 1 (detection of a $C$ in a ncighboring stream), they reported only second glimpse. The content of the first glinpse in procedure 1 would correspond to a memory of the target $C$. Indecd, when the entire response distriburion of procedure I was normalized for comparison with the second glimpse distributions of procedure 2, the three distributions were seen to be essentially equivalent (Fig. 2e), which confirmed objectively what the subjects reported introspectively.

Decreasing the intensity of the outline square illustrated independent control of the rwo glimpse processes. A dim but clearly visible square left the first glimpse complere-

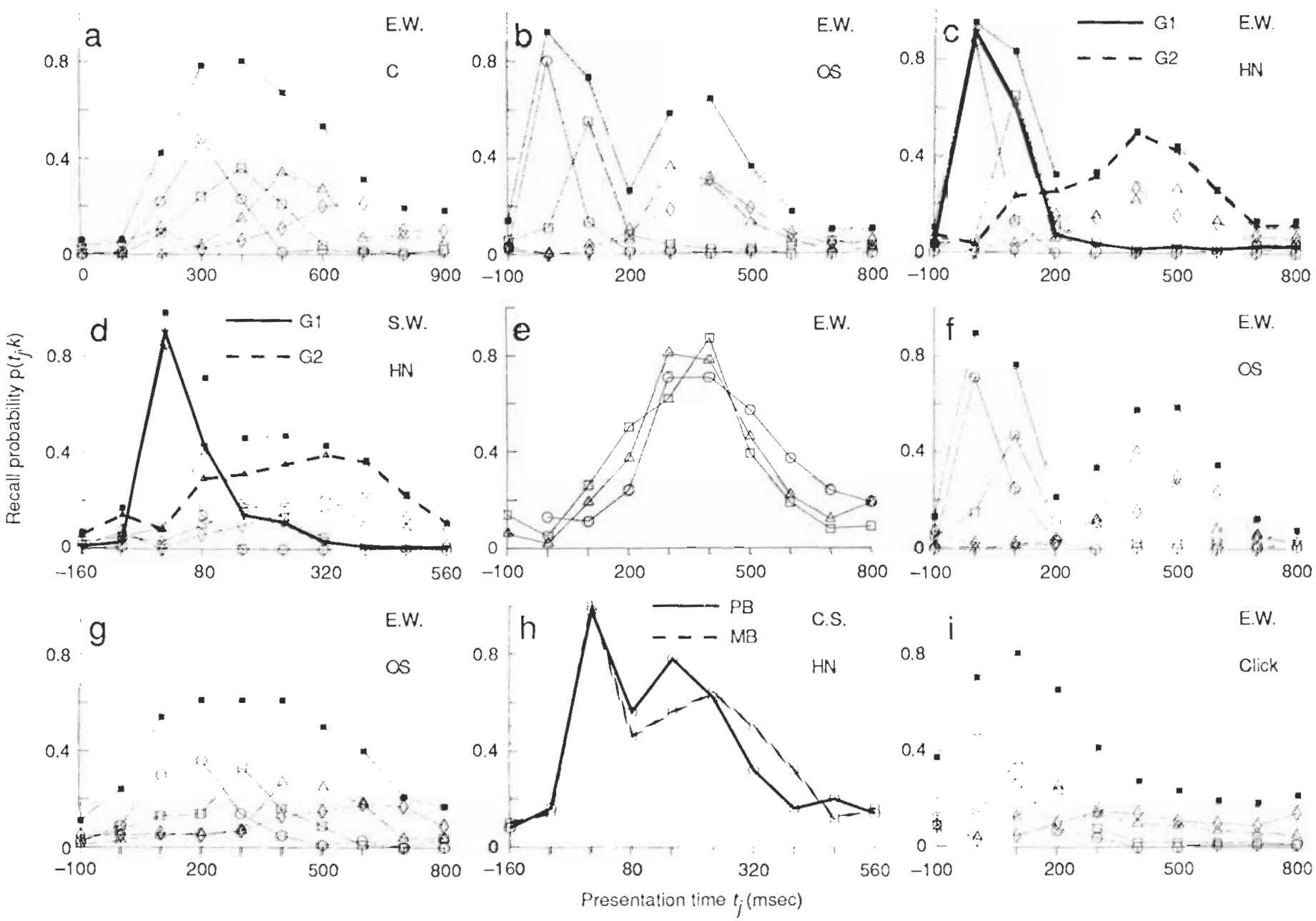

Fig. 2. Recall probability as a function of presentation tine of numerals from the numeral stream; the target occurs at tune 0 . The three subjects werc $\mathrm{F}_{\text {. W. }}$. $S . W$., and C.S. The envelope curve 1 describes the probability of recalling a numeral presented at time $t$ anywhere in the response sequence. In pancls a-d, $f$, $\mathrm{g}$, and $\mathrm{i}$, the inscribed curves give individual recall probabilities for cach of the four responses $\left[p\left(t_{j}, 1\right) 0, p\left(t_{j}, 2\right) \square, p\left(t_{j}, 3\right) \triangle, p\left(t_{j}, 4\right) \nabla\right]$, In pancis $i$ and $\mathrm{d}, \nabla$ and $\Delta$ represent glimpse I (Gl) and glimpse $2(G 2)$ data, respectively. In pancl e, the inscribed curves $O, \square$, and $\triangle$ are redefincd (O, C; $\square$, $H N ;$ and $\triangle, O S$ ). Numeral presentation rates were 10 per second unless otherwise noted. To better display the data, abscissas vary between panels. (a) Target $C$, procedure 1 . (b) Target outline square (OS), procedure 2. (c and d) Target highlighted numeral (HN), procedure 2 plus glimpse judgneme. The heivy solid and dashed lines indicate items jutiged to have occurred in GI and G2, respectively. The numeral presentation rate for S.W. Was 12.5 per second. (e) Comparison of data from procedure 1, targer C with G2 judgments from procedure 2, targets OS and HN. Curves are normalized to have the salme area. (f) Target dinumed OS. Sccond peak is shifted +100 msec relative to bright OS. (g) Further-dimuned target OS. First peak has been lost (GI). (h) Target HN, conteirison of recall in purc blocks (PB) with mixed blocks (MB) of HN and OS. (i) Target auditory click. 
ly intact but delayed the peak of the second glimpse by 100 msce (Fig. 2f). Further dimming of the outline square (to an intensity level at which the square was still always detected but motor reaction times were slowed by $65 \mathrm{msec}$ relative to the fullintensity square) greatly altered first glimpse responses (Fig. $2 \mathrm{~g}$ ). Interposing a bright masking field composed of a dense dot matrix between successive numeral frames also destroyed the first glimpse but left the second glimpse relatively intact. These two manipulations dissolved the perceptual glue (16) binding the outline square to the interior numeral.

In the case of the highlighted numeral target, when we randomly varied the intensiry of all the other numerals from frame to frame, and we made the highlighted numcral only somewhat more intense than the strongest of its competitors (a signal-tonoise manipulation), the first glimpse remaincd intact, but the second glimpse was delayed by about $100 \mathrm{msec}$. Another factor, level of practice, had strong effects on second glimpse responses but almost no effect on first glimpse responses.

Late in the stimulus sequence, before a target had occurred, the instantancous probabiliry that a target would occur became much greater than early in the sequence. Correspondingly, the subjects' expectancy of a target increased as the end of the stimulus sequence was approached. Like motor-reaction times, second glimpse responses sped up with this increased expectancy; first glimpse responses were unaffected. Another form of expectancy occurred in mixed-list stimulus presentations when the subject did not know which target to expect: OS or HN. Compared with purc blocks of OS or HN trials, the second glimpse mixed-list responses were later in the numeral sequence; the first glimpse responses were unaffected by this form of stimulus uncertainty (Fig. 2h).

Wc also used an auditory click as the stimulus for triggering an attentional gate. The distribution of numerals reported in response to the click stimulus is shown in Fig. 2i. The cross-modality data showed an appreciable proportion of recalls of the nutmeral that preceded the target by $100 \mathrm{msec}$. These early recalled stimuli indicated that, in the absence of competing visual stimuli, there was some memory for visual stimuli that preceded the attention trigger; they also indicated that an intermodality attention shift could be extremely quick. The click procedure demonstrated the feasibility of directly measuring the gating of visual inputs by auditory signals, a critical featurc of the partial report procedure for measuring visual very short term memory (2) and of the Reeves-Sperling theory of temporal order judgments ( $I$ ).

Bimodal distributions of reports from a rapid numeral stream arc explained in terms of two consecurive, partially overlapping artentive processes. A fast process (interpreted as a first glimpse by the subject) occurred automatically upon target detection and resulted in almost perfect recall of the target numeral as well as in occasional recall of a numeral immediately after the target. The autematic process was effortless, required no practice, and was virtually independent of task difficulty. For the subject to have recalled a numeral from the numeral stream in the auromatic process required that his or her attention was not withdrawn to another location away from numerals (as in procedure 1) and that the target was sufficicntly distinctive (for example, not too dim). With outline square targets (but not with highlighted numeral targets), the automatic process was destroyed by masking ficlds interposed between successive numeral frames.

An effortful, slow attentive process (interpreted as a second glimpse by the subject) mediated recall of numerals occurring more than 200 to $300 \mathrm{msec}$ after the target. This was a controlled attentional process whose latency depended on factors that classically affect task difficulty: practice, expectation, stimulus probability in mixed-list designs, and target signal-to-noise ratio.
REFERENCES AND NOTES

1. A. Reeves and G. Sperling, Psychol. Rcr, 93, 180 (1986).

2. G. Spurling, Psychol Monogr. 74, No. 11 (whole No. 498) (1960)

3. M. Colthcart, Percept. Psychoplys. 27, 183 (1980).

4. G. M. Long, Prychol. Bull. 88, 785 (1980).

5. M. L. Shaw and P. Shaw, /. Exp. Psychol: Human Percept. Perfonm. 3, 201 (1977).

6. I. A Swets, in Varietios of Ancmion, R. Parasuraman and D. R. Davies, Eds. (Academic Press, Now York, 1984), Pp. 183-242.

7. D. F. Broadbent, Psychol. Rev. 64, 205 (1957).

8. A. Treisman, ibid. 76. $282(1969)$.

9. R. M. Shiffrin, in Stcuins' Handhook of Experimental Psyclology, R. C. Atkirson, R. J. Herrnstein, G. I.indzey, R. D. Luce, Eds. (Wiley, New York, cd. 2, in press).

10. G. Spcrling and B. A. Dosluer, in Handbook of Perception and Penformance. K. Bot, L. Kaufman, J. Thomas, Eds. (Wiley, New York, 1986), vol. 1, pp. 2.1-2.65.

11. E. Weichsclgartncr, thesis, New York University, (1984).

12. R. M. Shiffrin and W. Schncider, Psychol. Rev. 84, 127 (1977).

13. G. Sperling and A. Recres, in Atention and Perfomance, R. Nickerson, Ed. (Frlbaum, Hillsdale, NJ, 1980), vol. 8, pp. 347-360.

14. M. C. Potter and E. 1. Levy, J. Fxp. Psychol 81, 10 (1969).

15. G. Spurling, J. Budiansky, J. G. Spivak, M. C. Johnson, Scicnce 174, 307 (1971)

16. A. M. Treisman and G. Gelade, Cog. Psychol. 12, 97 (1980).

17. The cxperiments described herein were submitted by E.W. in partial fulfillment of the requirements fot the Ph, 1). degree from Now York Lniversity. The experimental work was performed at the Human Information Processing Laboratory, New York University, supported by U.S. Air Force Office of Scicntific Resciarh, life Science Directorate, grant $85-0364$.

7 May 1987; accepted 8 Scptomber 1987

\title{
Mechanism of the Body-Centered Cubic-Hexagonal Close-Packed Phase Transition in Iron
}

\author{
W. A. Bassett AND E. HuAng
}

The transition from body-centered cubic to hexagonal close-packed phase in iron has been studied in a diamond anvil cell with synchrotron radiation. The hexagonal closepacked phase, when it first appears, has a ratio of lattice parameters that is significantly larger than normal. This is attributed to a displacive mechanism that causes a distortion of the hexagonal close-packed structure in a body-centered cubic matrix. The hexagonal close-packed phase adjacent to a boundary with the body-centered cubic phase is stretched in the $c$ direction and compressed in the $a$ direction when it first forms.

A T ROOM TEMPERATURE AND ATMOspheric pressure, iron has the bodycentered cubic (bce) structure. The bcc phase of iron undergoes a polymorphic transition at high pressure that was first reported by Bancroft $e t$ al. (l). The structure of the high-pressure polymorph of iron was later identified as hexagonal close-packed (hep) (2). The bec-hcp phase transition has been studied by a number of techniques since it was first recognized (3-5). The reported transition pressure ranges from 5 to 15 GPa (6). In order to explain this scatter, it is important to understand the mechanisn of the phase transformation. A mechanism for the bec-hcp phase transition in iron was proposed by Mao et al. (7). Confirmation of their mechanism, however, has had to await the new capabilities of synchrotron radiation.

The high intensity of synchrotron radiation allows us to rapidly acquire diffraction

Department of Gcological Scicnces, Comell University, Ithaca, NY 14853 